\title{
Adherence to ESMO clinical recommendations for prophylaxis of chemotherapy-induced nausea and vomiting
}

\author{
Henning Burmeister • Stefan Aebi • Cristina Studer • \\ Martin F. Fey • Oliver Gautschi
}

Received: 23 August 2010 /Accepted: 27 December 2010/Published online: 14 January 2011

(C) Springer-Verlag 2011

\begin{abstract}
Background We assessed adherence to the European Society of Medical Oncology (ESMO)/Multinational Association of Supportive Care in Cancer recommendations for prophylaxis of chemotherapy-induced nausea and vomiting (CINV) at our institution.

Patients and methods The charts of 299 patients starting a new chemotherapy between November 2008 and April 2009 were reviewed. Baseline characteristics and prophylaxis of CINV during the first cycle were recorded, and adherence to ESMO recommendations was determined. Chi-square tests and logistic regression were used to test for predictors of adherence.

Results Prophylaxis of acute CINV was not adherent in 39\% of the patients: 39 of 54 patients with low emetogenic chemotherapy had a serotonin antagonist, and 24 of 100 with moderately emetogenic therapy had a neurokinin antagonist. Nevertheless, $71 \%$ of the patients treated with highly emetogenic therapy received the guideline-specified prescription. Prophylaxis of delayed CINV was not adherent in $89 \%$ of the patients: 101 of 125 patients with highly or moderately emetogenic single-day chemotherapy received a serotonin antagonist. Male gender (odds ratio (OR) 0.484, 95\% confidence interval (CI) $0.291-0.806 ; P=0.005$ ) and hematologic neoplasia (OR 2.151, 95\% CI 1.19-3.887; $P=0.011$ ) were independent predictors of non-adherence. Age (OR $0.981,95 \%$ CI $0.964-0.998 ; P=0.029)$ and inpatient treatment (OR $0.457,95 \%$ CI $0.25-0.836 ; P=0.011$ ) indicated a lower risk of non-adherence.
\end{abstract}

H. Burmeister $(\bowtie) \cdot$ S. Aebi $\cdot$ C. Studer $\cdot$ M. F. Fey $\cdot$ O. Gautschi Department of Medical Oncology, University Hospital Bern, 3010 Bern, Switzerland

e-mail: henning.burmeister@insel.ch
Conclusion Contrary to older studies reporting frequent omissions of corticosteroids, the current study demonstrated significant overuse of serotonin antagonists for prophylaxis of delayed CINV.

Keywords Chemotherapy · Nausea $\cdot$ Emesis $\cdot$ Supportive care $\cdot$ Guidelines $\cdot$ Corticosteroids

\section{Introduction}

Medical guidelines are generally developed to define therapeutic standards resulting in the optimization of treatment. Ideally, they should be evidence-based, economical, and practicable and should rapidly adjust to medical progress. Chemotherapy-induced nausea and vomiting (CINV) can be prevented efficiently by corticosteroids, serotonin and neurokinin antagonists, dopamine antagonists, and benzodiazepines [1-5]. Although generally well tolerated, these drugs are associated with potential side effects and interactions. Adverse effects of corticosteroids are well-known, including infection, hypertension, diabetes, edema, gastritis, psychosis, and many more. Serotonin antagonists frequently cause headache, dizziness, constipation, abdominal pain, and fatigue. The neurokinin antagonist aprepitant is a substrate and inhibitor of CYP3A4 and can interact with cytostatic agents, corticosteroids, coumarins, oral contraceptives, and other drugs. Serotonin and neurokinin antagonists are associated with significant expense. To foster adequate prophylaxis of CINV, many organizations have published guidelines and recommendations, including the Multinational Association of Supportive Care in Cancer (MASCC), the American Society of Clinical Oncology (ASCO), the National Comprehensive Cancer Network, the European Society of Medical Oncology (ESMO), and others [6-12]. 
The ESMO clinical recommendations are the basis for the ESMO examination in medical oncology, which is required for board certification in most European countries, including Switzerland. ESMO recommendations are updated periodically and published in the Annals of Oncology.

The literature on the adherence to guidelines for prophylaxis of CINV in clinical practice is limited. Only few studies addressed this issue, and the results suggest that guidelines are difficult to transfer to the clinic [13-17]. Therefore, we assessed the adherence to the ESMO recommendations at our institution.

\section{Methods}

The study was approved by the Research Ethics Committee of the Canton of Berne, Switzerland. Using the electronic patient management system, we retrospectively identified all patients who received chemotherapy for the first time at our institution between November 1, 2008 and April 30, 2009. We collected the relevant baseline characteristics from the patient records, including patient age, gender, cancer diagnosis, chemotherapy, therapeutic intent, inpatient or outpatient setting, concomitant radiotherapy, and board certification of the treating physician. From the written and electronic prescription documents, we collected all the prophylactic orders for acute and delayed CINV, administered by oral or intravenous route during the first cycle of chemotherapy. Doses and schedules of neurokinin antagonists, serotonin antagonists, dopamine antagonists, benzodiazepines, and corticosteroids were recorded. The expected time course of CINV was categorized as follows: acute emesis within $24 \mathrm{~h}$ after administration of chemotherapy, delayed emesis arising $24 \mathrm{~h}$ after chemotherapy or later, and anticipatory emesis occurring days or hours before the beginning of chemotherapy. The ESMO/MASCC clinical recommendations cover all three categories. In this study, anticipatory CINV was not relevant because only the beginning of first cycle was investigated. Chemotherapies were divided into four groups according to their emetogenic potential: high risk $(>90 \%$ patients suffer from nausea/vomiting without prophylaxis), moderate risk (30-90\%), low risk (10-30\%), and minimal risk $(<10 \%)$. Adherence to the 2008 ESMO recommendations was determined for each patient [10]. Adherence failure was defined (a) if a recommended antiemetic was not prescribed, (b) if the recommended dose level was not reached, or (c) if a non-recommended antiemetic was prescribed. Established dexamethasone dose equivalents for methylprednisolone were used [18]. Patient characteristics and treatment details were summarized by standard descriptive methods. Predictors of non-adherence with guidelines were investigated by univariate logistic regression analysis; significant covariates in univariate analysis were entered into a multivariate logistic regression model.

\section{Results}

Baseline characteristics Between November 2008 and April 2009, 299 consecutive patients started a new chemotherapy at our institution (Table 1). The median age was 60 years, $178(60 \%)$ were male, and $140(47 \%)$

Table 1 Baseline characteristics

\begin{tabular}{|c|c|c|}
\hline & Absolute & Percent $^{\mathrm{a}}$ \\
\hline Total & 299 & 100 \\
\hline \multicolumn{3}{|l|}{ Age } \\
\hline Median (years) & 60 & \\
\hline Range (years) & $19-93$ & \\
\hline \multicolumn{3}{|l|}{ Gender } \\
\hline Male & 178 & 60 \\
\hline Female & 121 & 40 \\
\hline \multicolumn{3}{|l|}{ Setting } \\
\hline Outpatients & 159 & 53 \\
\hline Inpatients & 140 & 47 \\
\hline \multicolumn{3}{|l|}{ Diagnosis } \\
\hline Solid cancer & 216 & 72 \\
\hline Hematologic malignancies & 83 & 28 \\
\hline \multicolumn{3}{|l|}{ Intention of chemotherapy } \\
\hline Curative & 164 & 55 \\
\hline Palliative & 135 & 45 \\
\hline \multicolumn{3}{|l|}{ Line of chemotherapy } \\
\hline First line & 267 & 89 \\
\hline Further lines & 32 & 11 \\
\hline \multicolumn{3}{|l|}{ Emetogenic potential } \\
\hline High & 97 & 32 \\
\hline Moderate & 100 & 33 \\
\hline Low & 54 & 18 \\
\hline Minimal & 48 & 16 \\
\hline \multicolumn{3}{|l|}{ Duration of chemotherapy } \\
\hline Single day & 148 & 49 \\
\hline Several days & 151 & 51 \\
\hline \multicolumn{3}{|l|}{ Concomitant radiation } \\
\hline No & 217 & 73 \\
\hline Yes & 81 & 27 \\
\hline \multicolumn{3}{|l|}{ Treating physician } \\
\hline Board certified (10) & 119 & 40 \\
\hline In training (9) & 180 & 60 \\
\hline
\end{tabular}

${ }^{\mathrm{a}}$ Sum can deviate from $100 \%$ because of rounding 
were inpatients. Solid cancers were present in $216(72 \%)$ patients and hematologic malignancies in $83(28 \%)$ patients. Two hundred sixty-seven $(89 \%)$ patients received first-line chemotherapy, and $32(11 \%)$ patients received further lines of chemotherapy. The emetogenic potential of chemotherapy was high in $97(32 \%)$ patients, moderate in 100 (33\%; including eight patients with doxorubicin and cyclophosphamide), low in $54(18 \%)$, and minimal in 48 $(16 \%)$ patients. The most frequent therapies were cisplatin or 5-fluorouracil (with concomitant radiation), followed by gemcitabine, bortezomib, and rituximab, cyclophosphamide, doxorubicin, vincristine, and prednisone. The duration of chemotherapy was single day in 148 (49\%) and several days in $151(51 \%)$ patients. One hundred nineteen $(40 \%)$ patients were treated by a board-certified physician, and $180(60 \%)$ patients were treated by a physician in medical oncology training supervised by a board-certified physician.

Adherence to ESMO/MASCC recommendations Overall, prophylaxis of acute CINV was compliant in 181 of 299 (61\%) patients and non-compliant in 118 (39\%) patients (Fig. 1). Prescription of neurokinin antagonists, serotonin antagonists, and corticosteroids for prophylaxis of acute CINV was compliant in $83 \%, 82 \%$, and $85 \%$, respectively. Prophylaxis of delayed CINV, assessed in the 148 patients receiving single-day chemotherapy, was compliant in 17 of 148 (11\%) patients and noncompliant in 131 (89\%) patients. Prescription of neurokinin antagonists, serotonin antagonists, and corticosteroids for prophylaxis of delayed CINV was compliant in $70 \%, 11 \%$, and $61 \%$, respectively. Dopamine agonists and benzodiazepines were almost exclusively prescribed as on-demand medication; they were therefore excluded from further analysis.
In the highly emetogenic potential group, 65 patients had a single-day chemotherapy, and 32 patients received chemotherapy over several days (Table 3). Prophylaxis of acute CINV on day 1 was adherent in 69 of $97(71 \%)$ patients, whereas 28 of 97 (29\%) patients did not receive adherent prophylaxis, mainly caused by incorrect use of aprepitant. The overuse of a triple antiemetic strategy including a neurokinin antagonist, serotonin antagonist, and corticosteroids was observed in 10 of 32 patients undergoing high-risk chemotherapy over several days, whereas 6 of 32 patients were treated insufficiently without a neurokinin antagonist. In this subgroup, five patients received procarbazine orally. In single-day treatment, all three patients on dacarbazine and two of three patients on doxorubicin, bleomycin, vinblastine, and dacarbazine (ABVD) were undertreated without a neurokinin antagonist. Adherence to recommendations for prophylaxis of delayed CINV on days 2 and 3 was poor (20\% and $35 \%$, respectively) because 59 of 65 patients with single-day chemotherapy received a serotonin antagonist for several days. The prescription of corticosteroids was generally compliant, except that the recommended dose reduction in combination with aprepitant was frequently not followed. Adherence on days 4 and 5 was fair ( $76 \%$ and $88 \%$, respectively).

In the moderately emetogenic potential group, 60 patients had a single-day chemotherapy, and 40 patients received chemotherapy over several days (Table 4). Prophylaxis of acute CINV on day 1 was compliant in 66 of $100(66 \%)$ patients. Twenty-two patients received a neurokinin antagonist (14 patients with single-day chemotherapy and eight with chemotherapy over several days). Half of the patients treated incorrectly with a neurokinin antagonist had hematologic malignancies and were treated as inpatients. Seven patients were insufficiently treated on day 1 , five of them because corticosteroids were omitted.
Fig. 1 Adherence over time and emetogenic potential. $C S$ corticosteroid, HT 5hydroxytryptamine (serotonin) antagonist, $N K$ neurokinin antagonist, $D 1-5$ days $1-5$

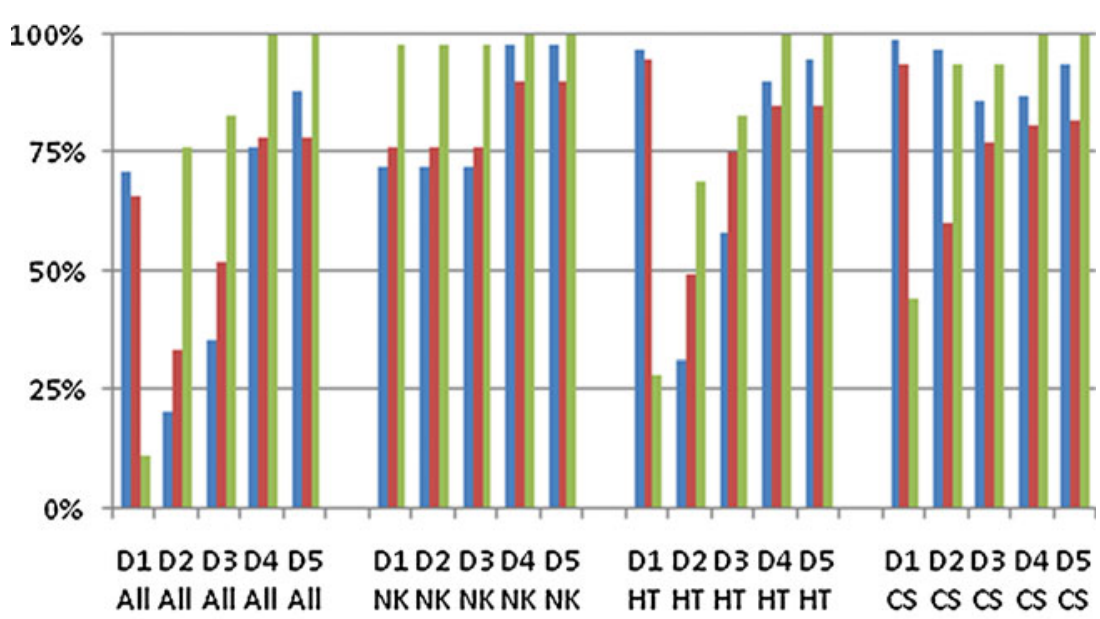

- High $(\mathrm{N}=97) \quad$ Moderate $(\mathrm{N}=100) \quad$ Low $(\mathrm{N}=54)$ 
Adherence to recommendations for prophylaxis of delayed CINV was poor (33\% and 52\%) mainly because 42 of 60 patients with single-day chemotherapy received a serotonin antagonist on day 2 and 21 of 60 patients still on day 3 additionally to corticosteroids. On the contrary, patients receiving temozolomide orally in a multiday regime were undertreated in $56 \%$ of the cases, since they did not receive corticosteroids as recommended. Adherence on days 4 and 5 was fair (78\% and 78\%). Among the eight patients in the moderate group on doxorubicin and cyclophosphamide, six had compliant prophylaxis and two patients had no neurokinin antagonist because a neurokinin antagonist was considered optional by the internal guidelines of the gynecological oncology unit. All patients given the combination of adriamycin and cyclophosphamide inappropriately received an additional prescription of a serotonin antagonist for delayed emesis.

Adherence to guidelines in the low emetogenic potential group was only 6 of $54(11 \%)$, due to the noncompliant combination of a serotonin antagonist in addition to corticosteroids in 39 patients (Table 5). Seventeen of $54(24 \%)$ patients in this group received a serotonin antagonist against delayed emesis on day 2 . Nine patients were still treated with this medication on day 3 , and one patient received a neurokinin antagonist. Nine patients received no antiemetics. In the minimally emetogenic potential group, adherence was 40 of 48 (83\%; data not shown). Six patients received vinorelbine for peripheral stem cell mobilization, plus a serotonin antagonist for prophylaxis of acute CINV, as prescribed by the internal guidelines of the autologous transplant unit.

Overall, 81 of 299 (27\%) patients had concomitant radiotherapy, including 36 patients on cisplatin. Although radiotherapy by itself can be emetogenic, the 2008 ESMO recommendations did not address this issue specifically. Out of this group, one patient with moderately emetogenic chemotherapy was overtreated by receiving a neurokinin antagonist, whereas four patients with highly emetogenic chemotherapy did not receive any neurokinin antagonist. All 26 patients with radiotherapy and concomitant minimally emetogenic chemotherapy correctly received no prophylaxis.

Predictors of non-adherence Logistic regression was used to identify factors associated with adherence to ESMO/ MASCC clinical recommendations for prophylaxis of CINV (Table 2). In the univariate analysis, low and moderate emetogenic potential predicted non-adherence. On the contrary, higher age, solid tumor type, and inpatient were associated with better adherence. In the multivariate analysis, younger age was associated with poor adherence, whereas hematologic neoplasia, men, and outpatient were twice at risk of non-adherence. High emetogenic potential was found to be tightly correlated with inpatient treatment. Only 16 of 97 patients receiving highly emetogenic chemotherapy were outpatients. Therefore, emetogenic potential was not an independent predictor of adherence in the multivariate analysis.

\section{Discussion}

Our study showed that during the study period, adherence to ESMO/MASCC recommendations for prophylaxis of CINV at our institution was not optimal. The major deviation from the 2008 ESMO clinical recommendations was an overuse of serotonin antagonists for prophylaxis of acute CINV in patients with low emetogenic chemotherapy and overuse of serotonin antagonists for prophylaxis of delayed CINV in patients with highly and moderately emetogenic chemotherapy. A clear differentiation between standby medication and fixed prescription was not recognizable, and it is therefore possible that our study overestimated the use of serotonin antagonists for prophylaxis of delayed CINV. In some patients with moderately emetogenic chemotherapy, overuse of neurokinin antagonists was also noted. Although not technically compliant with the ESMO guidelines, the treatment received by these patients cannot be regarded as inadequate as serotonin antagonists are likely to be effective with low emetogenic chemotherapy. In their recent study, Rapoport et al. also show the benefit of including neurokinin antagonists as part of the standard therapy for moderate emetogenic chemotherapy [19]. Most patients on aprepitant received corticosteroids at full doses, despite the recommended dose reduction to $50-75 \%$ in the 2008 ESMO recommendations [10]. In the updated 2009 ESMO recommendations, published in May 2009 after the registration period of our study, this specific point was deleted, and a total dexamethasone dose of $12 \mathrm{mg}$ was mentioned [11]. In 2010, a guideline update for MASCC and ESMO was published, including a comprehensive review of the literature and recommendations for prevention of radiotherapy-induced nausea and vomiting [12].

At our institution, adherence to ESMO recommendations in patients receiving bleomycin, etoposide, doxorubicin, cyclophosphamide, vincristine, procarbazine, and prednisone (BEACOPP) or ABVD chemotherapy was poor because only few of these patients received a neurokinin antagonist. Although procarbazine and dacarbazine are classified as highly emetogenic, our own experience supports the view that most patients receiving BEACOPP or ABVD do not experience CINV 
Table 2 Logistic model coefficients table for overall adherence

\begin{tabular}{|c|c|c|c|c|c|c|}
\hline & \multicolumn{3}{|c|}{ Univariate analysis } & \multicolumn{3}{|c|}{ Multivariate logistic regression analysis } \\
\hline & OR & RR $95 \%$ CI & $P$ (Wald) & OR & OR $95 \% \mathrm{CI}$ & $P$ (Wald) \\
\hline Age & 0.982 & $0.966-0.998$ & 0.027 & 0.981 & $0.964-0.998$ & 0.029 \\
\hline Gender (female vs. male) & 0.496 & $0.304-0.808$ & 0.005 & 0.484 & $0.291-0.806$ & 0.005 \\
\hline Physician (non-certified vs. certified) & 1.062 & $0.662-1.705$ & 0.802 & & & \\
\hline Emetogenic potential (high vs. low + moderate) & 0.505 & $0.3-0.849$ & 0.009 & 0.766 & $0.392-1.497$ & 0.436 \\
\hline Tumor type (hematologic vs. solid) & 1.829 & $1.097-3.049$ & 0.020 & 2.151 & $1.19-3.887$ & 0.011 \\
\hline Inpatient vs. outpatient & 0.496 & $0.309-0.799$ & 0.004 & 0.457 & $0.25-0.836$ & 0.011 \\
\hline
\end{tabular}

$O R$ odds ratio, $R R$ relative risk, $C I$ confidence interval

when the neurokinin antagonist is omitted. Future recommendations should include more information on evidence-based indications for neurokinin antagonists versus situations where the benefit is less well-known.

Focusing on the highly emetogenic group, we found out that more than $70 \%$ of patients were treated correctly by receiving a triple regime: a neurokinin antagonist, a serotonin antagonist, and corticosteroids. The lack of adherence on day 2 was primarily due to the prescription of a serotonin antagonist, which in this setting probably contributes minimally to antiemetic control. Nevertheless, these results contrast with two older studies from the USA, reporting frequent omission of corticosteroids to prevent delayed CINV. The study by De Angelis et al. was published as an abstract, and the study by Mertens et al. was published in a peerreviewed journal $[15,16]$. Mertens et al. assessed the prophylaxis of CINV in 125 patients with highly emetogenic chemotherapy. According to internal guidelines, based on the 1999 ASCO recommendations, 73\% of the patients in the Mertens study did not receive adequate prophylaxis [20]. Only $25 \%$ of patients correctly received corticosteroids for prophylaxis of delayed CINV, and $64 \%$ of the patients experienced nausea during the first 5 days. Our study, however, showed frequent overuse of antiemetics for CINV prophylaxis. This could be clinically and economically relevant. Although we collected data from a large set of patients, our study was limited because it was unicentric and retrospective. However, our results are very consistent with the study by Roila et al. [21], which reported on the adherence in 77 Italian centers. Although the guidelines and some of the prophylactic medications have been modified since 2000 , there still remains undertreatment in acute prophylaxis in the high-risk group and overtreatment in acute and delayed prophylaxis in the moderate-, low-, and minimal-risk groups. We encourage other centers to conduct similar studies, to shed more light on the magnitude of the problem.
An important question arising from our study is how to improve the current situation, since adherence to the guideline is related with a better control of CINV. In a cohort study, O'Kane shows that adhering to MASCC antiemetic guidelines significantly reduces CINV with cisplatin and oxaliplatin [22]. Our results suggest that adherence was not related to board certification of the treating physician. All except one board-certified medical oncologist at our institution had previously taken the ESMO exam. All physicians in training had previously undergone periodical education in supportive care and were supervised by board-certified physicians. Our study emphasizes the need for continued education. However, Mertens et al. demonstrated that education alone did not substantially improve adherence [16]. In their study, physician adherence was enhanced when delayed CINV occurred due to omission of prophylaxis. Symptoms specifically related to an overuse of antiemetics (for instance, constipation, dizziness, or fatigue) such as pointed out in our study fail to play this part, since they can also be caused by cancer itself or by chemotherapy. Underuse of antiemetics will predictably lead to patient reports and complaints at follow-up visits, and such patient-reported outcome measures have been shown to be effective in changing the prescription practice of physicians. Patients reporting their symptoms in a selfmanaged diary could improve symptom control and patient compliance. Overprescription of antiemetics is, however, much less likely to be detected by patients, since overuse can only be detected if in patients without CINV a given regime of antiemetics is deliberately tapered to the minimally required dosage level to prevent this type of side effect. In clinical practice, both patients and physicians are somewhat reluctant to finetune the dosage of antiemetics during follow-up visits in this manner, and successful albeit overdosed CINV prophylaxis is likely to be maintained. Other studies have shown that pharmacy- or nursing-controlled prescriptions and software ordering tools can enhance 
adherence $[13,23]$. As a consequence, we are currently implementing software-based prophylaxis of CINV at our institution, to reduce overuse and potential harm in our patients. This software automatically adds the appropriate antiemetics to the chemotherapy which is prescribed. Accreditation programs, including the ESMO program for the Integration of Oncology and Palliative Care, may offer a chance to introduce such measures to a broader community [24].

Acknowledgments The authors would like to thank Daniel Ratschiller for database management. An acknowledgment should also go to Jürg Bernhard for his comments.

Potential conflicts of interest The authors declare no conflicts of interest.

\section{Appendix}

Table 3 Adherence in patients with highly emetogenic chemotherapy

\begin{tabular}{|c|c|c|c|c|c|c|}
\hline \multirow[b]{2}{*}{ Day 1} & \multicolumn{2}{|c|}{$\begin{array}{l}\text { Total } \\
(N=97)\end{array}$} & \multicolumn{2}{|c|}{$\begin{array}{l}\text { Single-day } \\
(N=65)\end{array}$} & \multicolumn{2}{|c|}{$\begin{array}{l}\text { Several days } \\
(N=32)\end{array}$} \\
\hline & & & & & & \\
\hline - overall & 69 & $71 \%$ & 53 & $82 \%$ & 16 & $50 \%$ \\
\hline - NK & 70 & $72 \%$ & 54 & $83 \%$ & 16 & $50 \%$ \\
\hline - HT & 94 & $97 \%$ & 64 & $98 \%$ & 30 & $94 \%$ \\
\hline - CS & 96 & $99 \%$ & 65 & $100 \%$ & 31 & $97 \%$ \\
\hline \multicolumn{7}{|l|}{ Day 2} \\
\hline - overall & 19 & $20 \%$ & 6 & $9 \%$ & 13 & $41 \%$ \\
\hline - NK & 70 & $72 \%$ & 54 & $83 \%$ & 16 & $50 \%$ \\
\hline - HT & 30 & $31 \%$ & 6 & $9 \%$ & 24 & $75 \%$ \\
\hline - CS & 94 & $97 \%$ & 62 & $95 \%$ & 32 & $100 \%$ \\
\hline \multicolumn{7}{|l|}{ Day 3} \\
\hline - overall & 34 & $35 \%$ & 22 & $34 \%$ & 12 & $38 \%$ \\
\hline - NK & 70 & $72 \%$ & 54 & $83 \%$ & 16 & $50 \%$ \\
\hline - HT & 56 & $58 \%$ & 34 & $52 \%$ & 22 & $69 \%$ \\
\hline - CS & 83 & $86 \%$ & 51 & $78 \%$ & 32 & $100 \%$ \\
\hline \multicolumn{7}{|l|}{ Day 4} \\
\hline - overall & 74 & $76 \%$ & 55 & $85 \%$ & 19 & $59 \%$ \\
\hline - NK & 95 & $98 \%$ & 65 & $100 \%$ & 30 & $94 \%$ \\
\hline - HT & 87 & $90 \%$ & 62 & $95 \%$ & 25 & $78 \%$ \\
\hline - CS & 84 & $87 \%$ & 57 & $88 \%$ & 27 & $84 \%$ \\
\hline \multicolumn{7}{|l|}{ Day 5} \\
\hline - overall & 85 & $88 \%$ & 65 & $100 \%$ & 20 & $63 \%$ \\
\hline - NK & 95 & $98 \%$ & 65 & $100 \%$ & 30 & $94 \%$ \\
\hline - HT & 92 & $95 \%$ & 65 & $100 \%$ & 27 & $84 \%$ \\
\hline - CS & 91 & $94 \%$ & 65 & $100 \%$ & 26 & $81 \%$ \\
\hline
\end{tabular}

CS corticosteroid; HT 5-hydroxytryptamine (serotonin) antagonist; $N K$ neurokinin antagonist
Table 4 Adherence in patients with moderately emetogenic chemotherapy

\begin{tabular}{|c|c|c|c|c|c|c|}
\hline & \multicolumn{2}{|c|}{$\begin{array}{l}\text { Total } \\
(N=100)\end{array}$} & \multicolumn{2}{|c|}{$\begin{array}{l}\text { Single-day } \\
(N=60)\end{array}$} & \multicolumn{2}{|c|}{$\begin{array}{l}\text { Several days } \\
(N=40)\end{array}$} \\
\hline \multicolumn{7}{|l|}{ Day 1} \\
\hline - overall & 66 & $66 \%$ & 42 & $70 \%$ & 24 & $60 \%$ \\
\hline - NK & 76 & $76 \%$ & 44 & $73 \%$ & 32 & $80 \%$ \\
\hline - HT & 95 & $95 \%$ & 58 & $97 \%$ & 37 & $93 \%$ \\
\hline - CS & 94 & $94 \%$ & 59 & $98 \%$ & 35 & $88 \%$ \\
\hline \multicolumn{7}{|l|}{ Day 2} \\
\hline - overall & 33 & $33 \%$ & 14 & $23 \%$ & 19 & $48 \%$ \\
\hline - NK & 76 & $76 \%$ & 44 & $73 \%$ & 32 & $80 \%$ \\
\hline - HT & 49 & $49 \%$ & 18 & $30 \%$ & 31 & $78 \%$ \\
\hline$-\mathrm{CS}$ & 60 & $60 \%$ & 34 & $57 \%$ & 26 & $65 \%$ \\
\hline \multicolumn{7}{|l|}{ Day 3} \\
\hline - overall & 52 & $52 \%$ & 28 & $47 \%$ & 24 & $60 \%$ \\
\hline - NK & 76 & $76 \%$ & 44 & $73 \%$ & 32 & $80 \%$ \\
\hline - HT & 75 & $75 \%$ & 39 & $65 \%$ & 36 & $90 \%$ \\
\hline$-\mathrm{CS}$ & 77 & $77 \%$ & 46 & $77 \%$ & 31 & $78 \%$ \\
\hline \multicolumn{7}{|l|}{ Day 4} \\
\hline - overall & 78 & $78 \%$ & 50 & $83 \%$ & 28 & $70 \%$ \\
\hline - NK & 90 & $90 \%$ & 52 & $87 \%$ & 38 & $95 \%$ \\
\hline - HT & 85 & $85 \%$ & 50 & $83 \%$ & 35 & $88 \%$ \\
\hline - CS & 81 & $81 \%$ & 50 & $83 \%$ & 31 & $78 \%$ \\
\hline \multicolumn{7}{|l|}{ Day 5} \\
\hline - overall & 78 & $78 \%$ & 49 & $82 \%$ & 29 & $73 \%$ \\
\hline - NK & 90 & $90 \%$ & 52 & $87 \%$ & 38 & $95 \%$ \\
\hline - HT & 85 & $85 \%$ & 50 & $83 \%$ & 35 & $88 \%$ \\
\hline - CS & 82 & $82 \%$ & 51 & $85 \%$ & 31 & $78 \%$ \\
\hline
\end{tabular}

CS corticosteroid; HT 5-hydroxytryptamine (serotonin) antagonist; $N K$ neurokinin antagonist

Table 5 Adherence in patients with low emetogenic chemotherapy

Total $(\mathrm{N}=54)$

Day 1

- overall $6 \quad 11 \%$

- NK $\quad 53 \quad 98 \%$

- HT $15 \quad 28 \%$

- CS $24 \quad 44 \%$

Day 2

- overall $\quad 37 \quad 69 \%$

- NK $\quad 53 \quad 98 \%$

- HT $37 \quad 69 \%$

- CS $\quad 51 \quad 94 \%$

Day 3

CS corticosteroid; HT 5-

- overall $\quad 45 \quad 83 \%$

- NK $\quad 53 \quad 98 \%$

- HT $\quad 45 \quad 83 \%$

- CS $\quad 51 \quad 94 \%$ 
Table 6 Non-compliance subdivided into undertreated vs. overtreated

\begin{tabular}{lllll}
\hline & Compliant & Undertreated & Overtreated & Total \\
\hline Day 1 & & & & \\
- high & 69 & 18 & 10 & 97 \\
- moderate & 66 & 10 & 24 & 100 \\
- low & 6 & 9 & 39 & 54 \\
- minimal & 40 & 0 & 8 & 48 \\
Day 2 & & & & \\
- high & 19 & 15 & 63 & 97 \\
- moderate & 33 & 11 & 56 & 100 \\
- low & 37 & 0 & 17 & 54 \\
- minimal & 48 & 0 & 0 & 48 \\
Day 3 & & & & \\
- high & 34 & 23 & 40 & 97 \\
- moderate & 52 & 12 & 36 & 100 \\
- low & 45 & 0 & 9 & 54 \\
- minimal & 48 & 0 & 0 & 48 \\
\hline
\end{tabular}

\section{References}

1. Ioannidis JP, Hesketh PJ, Lau J (2000) Contribution of dexamethasone to control of chemotherapy-induced nausea and vomiting: a meta-analysis of randomized evidence. J Clin Oncol 18:3409-3422

2. Navari RM, Kaplan HG, Gralla RJ et al (1994) Efficacy and safety of granisetron, a selective 5-hydroxytryptamine-3 receptor antagonist, in the prevention of nausea and vomiting induced by high-dose cisplatin. J Clin Oncol 12:2204-2210

3. Roila F, Tonato M, Cognetti F et al (1991) Prevention of cisplatininduced emesis: a double-blind multicenter randomized crossover study comparing ondansetron and ondansetron plus dexamethasone. J Clin Oncol 9:675-678

4. Hesketh PJ, Grunberg SM, Gralla RJ (2003) The oral neurokinin1 antagonist aprepitant for the prevention of chemotherapyinduced nausea and vomiting: a multinational, randomized, double-blind, placebo-controlled trial in patients receiving highdose cisplatin - the aprepitant protocol 052 study group. J Clin Oncol 15:4112-4119

5. Gralla RJ, Itri LM, Pisko SE et al (1981) Antiemetic efficacy of high-dose metoclopramide: randomized trials with placebo and prochlorperazine in patients with chemotherapy-induced nausea and vomiting. N Engl J Med 305:905-909

6. The Antiemetic Subcommittee of the Multinational Association of Supportive Care in Cancer (MASCC) (2006) Prevention of chemotherapy- and radiotherapy-induced emesis: results of the 2004 Perugia International Antiemetic Consensus Conference. Ann Oncol 17:20-28

7. Gralla RJ, Roila F, Tonato M et al (2005) The 2004 Perugia Antiemetic Consensus Guideline process: methods, procedures, and participants. Support Care Cancer 13:77-79

8. American Society of Clinical Oncology (2008) Clinical practice guideline update: use of chemotherapy and radiation therapy protectants. J Clin Oncol 27(1):127-145
9. National Comprehensive Cancer Network (2006) Guidelines for supportive care, antiemesis. www.ncen.org

10. Herrstedt J, Roila F (2008) Chemotherapy-induced nausea and vomiting: ESMO clinical recommendations for prophylaxis. Ann Oncol 19:ii110-ii112

11. Herrstedt J, Roila F (2009) Chemotherapy-induced nausea and vomiting: ESMO clinical recommendations for prophylaxis. Ann Oncol 20:iv156-iv158

12. Roila F, Herrstedt J, Aapro M, ESMO/MASCC Guidelines Working Group et al (2010) Guideline update for MASCC and ESMO in the prevention of chemotherapy- and radiotherapyinduced nausea and vomiting: results of the Perugia consensus conference. Ann Oncol 21(Suppl 5):v232-v243

13. Nolte MJ et al (1998) Assuring the optimal use of serotonin antagonist antiemetics: the process for development and implementation of institutional antiemetic guidelines at Memorial Sloan-Kettering Cancer Center. J Clin Oncol 16(2):771778

14. The Italian Group for Antiemetic Research (1998) Transferability to clinical practice of the results of controlled clinical trials: the case of antiemetic prophylactic treatment for cancer chemotherapy-induced nausea and vomiting. Ann Oncol 9:759765

15. De Angelis V, Roila F, Patoia L et al (2000) Impact on antiemetic prescriptions of the Consensus Conference (CC) and of an expert's visit to oncological centers. Proc Am Soc Clin Oncol 19:606a, abstr

16. Mertens WC, Higby DJ, Brown D, Parisi R, Fitzgerald J, Benjamin EM, Lindenauer PK (2003) Improving the care of patients with regard to chemotherapy-induced nausea and emesis: the effect of feedback to clinicians on adherence to antiemetic prescribing guidelines. J Clin Oncol 21(7):13731378

17. Kaiser R (2005) Antiemetic guidelines: are they being used? Lancet Oncol 6:622-625

18. Axelrod L (2001) Corticosteroid therapy. In: Becker LB (ed) Principles and practice of endocrinology and metabolism, 3rd edn. Lippincott Williams \& Wilkins, Baltimore, pp 751-762

19. Rapoport B et al (2010) Aprepitant for the prevention of chemotherapy-induced nausea and vomiting associated with a broad range of moderately emetogenic chemotherapies and tumor types: a randomized, double-blind study. Support Care Cancer 18 (4):423-431

20. Gralla RJ, Osoba D, Kris MG et al (1999) Recommendations for the use of antiemetics: evidence-based, clinical practice guidelines. American Society of Clinical Oncology. J Clin Oncol 17:2971-2994

21. Roila F, De Angelis V, Patoia L et al (2000) Antiemetic prescriptions in 77 Italian oncological centers after MASCC consensus conference. Support Care Cancer 8:241

22. O'Kane (2009) Evaluate the effects of implementing the Multinational Association of Supportive Care in Cancer (MASCC) antiemetic guideline on the incidence of chemotherapy-induced nausea and vomiting (CINV) following platinum chemotherapy. Support Care Cancer 17:875

23. Teich JM, Merchia PR, Schmiz JL, Kuperman GJ (2000) Effects of computerized physician order entry on prescribing practices. Arch Intern Med 160:2741-2747

24. Cherny N et al (2010) European Society for Medical Oncology (ESMO) program for the integration of oncology and palliative care: a 5-year review of the designated centers' incentive program. Ann Oncol 21(2):362-369 